\title{
How to cope when becoming paralysed
}

JJ Wyndaele, Editor

Antwerp University Hospital Antwerp, Belgium

E-mail: wyndaelejj@skynet.be

It is a classical idea that an individual's response to becoming paralysed follows common patterns. But on the other hand it is also acknowledged that every individual will live such events in his very individual way.

When the impairment occurs suddenly, as after traumatic spinal cord injury, different stages of emotional response or psychological adjustment have been described. Those who work in a spinal unit can tell you they have witnessed disbelief, confusion, anger, grief, guilt or shame. What comes out in one person and why is subject to a lot of study. We want to try and understand in order to give a maximum of help, if required.

In this Spinal Cord issue we have several papers dealing with psychological aspects, quality of life and coping strategies. Peter et al. found in a large literature review that psychological resources were categorized into selfefficacy, self-esteem, sense of coherence, spirituality, optimism, intellect and other personality characteristics. Research looked often into associations of psychological resources with mental health and well-being, while associations with participation were rarely studied. Van Leeuwen et al., in another major literature review, found total locus of control, sense of coherence, self-worth, hope, purpose in life, and positive affect to be consistently associated with greater QOL. Negative affect and posttraumatic cognitions were consistently associated with lower QOL, while inconsistent evidence was found for subscales of LOC, the role of attribution of blame, spirituality, personality, appraisals, passive coping, and emotion-focused coping. No evidence was found for an association between active problem-focused coping styles and QOL. Augutis and Anderson specifically studied how adolescents who sustained SCI used a variety of strategies to help them cope with the consequences of the injury. They report how, as young adults in their own words, the coping strategies are recalled which they used as adolescents and how they conceptualized the process of coping. Krause and Bozard studied the natural course of changes in participation, employment, health, and subjective QOL over a 35-year interval after SCI. They found it marked by a survivor effect, where those with better outcomes were more likely to survive to follow up.

Recent papers on the subject coping-adjustment and QOL can be found in Spinal Cord 2010; 48: 598-602; Spinal Cord 2010; 48: 762-769; Spinal Cord 2011; 49: 362-370; Spinal Cord 2011; 49: 371-375 and Spinal Cord 2011; 49: 832-837.

Many more very interesting papers have been included dealing with other important subjects.

A small reminder that the Spinal Cord Prize is open till May 11 for competition as already mentioned in the editorial page of the 12th issue 2011.

Enjoy reading.

Spinal Cord (2012) 50, 173; doi:10.1038/sc.2012.15 